\title{
INSTRUMENTAL PARAMETERS OF THE U.S. NAVAL OBSERVATORY'S AUTOMATIC TRANSIT CIRCLE (ATC)
}

\author{
B. L. KLOCK and F. S. GAUSS \\ U.S. Naval Observatory, Washington D.C., U.S.A.
}

\begin{abstract}
The instrumental constants of the Naval Observatory's Automatic Transit Circle are discussed in conjunction with the transit circle's on-line data acquisition and control system. The reflecting collimators have been designed with CerVit primaries and minified point sources. The mark lens configuration is comprised of a long focal length lens and wedge manufactured from a single piece of fused silica. The on-line data acquisition and control system will permit real time monitoring of these instrumental parameters.
\end{abstract}

\section{Introduction}

The U.S. Naval Observatory's Automatic Transit Circle (ATC) is presently in its initial stage of performance evaluation of the instrumental constants. The automatic data acquisition for the constants is performed via a control program on an on-line IBM 1800 data acquisition and control system. The observer monitors the procedure from a controlled environment in the southeast corner of the transit circle pavilion. The only task that requires the observer to leave the control room is that of the alignment of the artificial sources in the north and south collimators. However, a method for automating this function is now under consideration.

\section{Instrumental Constants}

A detailed discussion of the optical system was presented (Klock, 1970) at the IAU 14th General Assembly. It shall suffice to mention here that an artificial source is located in the star tracker off the main optical axis by approximately 31 ".7 in $\alpha$, and 27.4 in $\delta$. This source is used in the conventional method of determining the level and nadir of the instrument with a mercury horizon.

The constants system presently has only one mark lens which is comprised of a long focal length $(116 \mathrm{~m})$ lens and wedge $\left(7^{\circ} 18^{\prime}\right)$ manufactured from a single piece of Schlieren quality fused silica. A new point source for the mark was designed and developed by H. E. Durgin, T. J. Rafferty, and R. J. Miller of the Observatory staff. The source is observed through a combination BG-18 and GG-400 Schott filter. The requirement for the wedge was generated by the close proximity of the collimator pier to the main instrument. The wedge-lens is mounted below the collimator at the end of the collimator pier closest to the telescope.

The collimator system encompasses two reflecting collimators with $25 \mathrm{~cm}$ diameter CerVit primaries and a novel self-contained artificial source. Each source has a range of motion of $\pm 6^{\prime \prime}$ in both the $x$ and $y$ coordinates. A star of approximately 4 th mag. is simulated. The actual diameter of the source is $0.9 \mathrm{~mm}$; however, the optical configuration minifies the source by a factor of 100 ; i.e., the apparent image size is

Gliese, Murray, and Tucker. 'New' Problems in Astrometry' 257 259. All Rights Reserved. Copyright o 1974 by the IAU 
$0.009 \mathrm{~mm}$. As an aid in alignment of one source apparently onto the other, the sources may be blinked alternately at a $15 \mathrm{~Hz}$ rate.

\section{Data Acquisition and Control Program}

As previously mentioned the ATC operates in conjunction with an IBM 1800 process control computer. The system has the capability to send information as well as control signals to the telescope, to receive data and status signals from the telescope, to communicate with the observer via a console typewriter, and to store $1 \frac{1}{2}$ million words of data on magnetic disk storage. Results can be printed on a line printer or punched onto cards.

As an aid to system checkout a program was written to allow each individual operation of the ATC to be controlled separately with the results displayed on a television monitor. The data registers can be loaded, read, and displayed in both binary and decimal formats.

The observational control program contains routines to compute the apparent place, to control the complete sequence of an observation, and to communicate with the observer. The latter consists of the issuance of messages concerning errors as well as the progress of the observation and of the receipt of information and commands from the observer. For example, the observer can request that the data be printed on the typewriter simultaneously with its storage on the disk and thus permit him to watch the progress of the observation.

The sequence of an observation begins with the entry of a star or constant number into a keyboard by the observer. He may enter star numbers one at a time or he may enter a list of up to 32 stars and constants. An entire night's observing list can also be stored for use on the disk.

If a star is to be observed, it is found on the disk file containing the 1950 rectangular coordinates. A second file contains the Besselian day numbers and precession matrix. The apparent place is then computed to an accuracy of approximately 0.01 ". The instantaneous refraction is calculated on the basis of the real time readings of the thermometer, barometer, and dew cell (all of which are automatically sampled by the computer). In the case of the constants the setting angle positions of the various light sources are stored. In order to follow the seasonal variations these settings are automatically corrected to the mean of the preceding night's observations.

Simultaneously with the preceding action the telescope is lifted hydraulically 0.05 $\mathrm{mm}$ and driven to the apparent declination, and then lowered. The telescope settles into a position within $\pm 15^{\prime \prime}$ of the desired position. The residual error between this position and the computed position is applied to the declination tracking mirror.

Once a star or constant source is detected and acquired for tracking the declination tracking mirror and right ascension tracking mirror are read out every second for thirty seconds on either side of the transit time for a star, and simply for sixty seconds for an instrumental constant. The computer continually monitors the tracking signals, makes decisions, and issues messages to the observer based on the length of 
time required to acquire the star and whether its signal is lost at any time (i.e., in the event of a cloud obscuration).

The data are stored on the disk for later reduction. However, certain calculations are performed in real time to allow the computer to check for error conditions. The temperature, pressure, and dew point are continually monitored and checked against similar backup systems. The speed at which certain operations occur is monitored to detect equipment failure. The pressure in the hydraulic lift mechanism is used to determine whether the telescope has settled onto the wyes before the readings are taken.

Mean values for the constants are computed, printed on the console typewriter, and automatically checked against the known range of values. The 1800 system has been used for checking errors in this manner on the six-inch transit circle for five years (Gauss, 1968) and has proven to be invaluable in detecting equipment failure and observer errors.

\section{Preliminary Results}

The expected accuracy of the ATC can be readily seen through the observational data collected with the constants. The internal repeatability of the tracking mirrors at the present time appears to be better than $0.1^{\prime \prime}$; for the main declination axis Inductosyn the value is slightly higher, $0.15^{\prime \prime}$.

\section{References}

Gauss, F. S.: 1969, Bull. Am. Astron. Soc. 1, 179.

Klock, B. L.: 1970, Trans. IAU XIVA, Report Commission 8. 